\title{
In patients suffering from idiopathic central serous chorioretinopathy, anxiety scores are higher than in healthy controls, but do not vary according to sex or repeated central serous chorioretinopathy
}

\author{
This article was published in the following Dove Press journal: \\ Neuropsychiatric Disease and Treatment \\ 28 April 2015 \\ Number of times this article has been viewed
}

\author{
Nooshin Bazzazi' \\ Mohammad Ahmadpanah ${ }^{2}$ \\ Siamak Akbarzadeh' \\ Mohammad Ali Seif Rabiei ${ }^{3}$ \\ Edith Holsboer-Trachsler ${ }^{4}$ \\ Serge Brand $d^{4,5}$ \\ 'Department of Ophthalmology, \\ Faculty of Medicine, Hamadan \\ University of Medical Sciences, \\ Hamadan, Iran; ${ }^{2}$ Research Center for \\ Behavioral Disorders and Substance \\ Abuse, Hamadan University of Medical \\ Sciences; Hamadan, Iran; ${ }^{3}$ Department \\ of Social Medicine, Faculty of \\ Medicine, Hamadan University \\ of Medical Sciences, Hamadan, Iran; \\ ${ }^{4}$ Psychiatric Clinics of the University \\ of Basel, Center for Affective, \\ Stress and Sleep Disorders, Basel, \\ Switzerland; ${ }^{5}$ Department of Sport \\ and Health Science, Sport Science \\ Section, University of Basel, Basel, \\ Switzerland
}

\begin{abstract}
Introduction: Idiopathic central serous chorioretinopathy (CSCR) is a relatively common ophthalmic disorder characterized by the development of a serous detachment of the sensory retina. Psychophysiological factors may trigger or maintain CSCR, though, surprisingly, the association between CSCR and anxiety has yet to be studied. The aims of the present study were threefold: to determine whether 1) Iranian patients with CSCR have higher scores for anxiety, 2) anxiety is lower, if CSCR has been experienced twice, and whether 3) anxiety scores differ between sexes.
\end{abstract}

Methods: A total of 30 patients with CSCR and 30 healthy age- and sex-matched controls took part in the study. A brief face-to-face interview was conducted covering demographic variables and history and occurrence of CSCR and assessing anxiety.

Results: Compared to healthy controls, anxiety was significantly higher in both first-time and second-time CSCR patients. In CSCR patients, anxiety scores did not differ between sexes.

Conclusion: Higher anxiety scores were observed in Iranian patients with CSCR, irrespective of whether this was the first or second occurrence of CSCR. This suggests there is no psychological adaptation in terms of reduced anxiety among patients with repeated CSCR.

Keywords: idiopathic central serous chorioretinopathy, anxiety, Iranian population, healthy controls

\section{Introduction}

Central serous chorioretinopathy (CSCR) is a relatively common ophthalmic disorder characterized by the development of a serous detachment of the sensory retina due to a deficient pumping function at the retinal pigment epithelium level. ${ }^{1-3}$ Diagnosis of CSCR is made using fluorescein angiography, while retinal optical coherence tomography can demonstrate shallow serous detachment. ${ }^{2,4}$ CSCR typically affects adults between 30 and 50 years of age. Symptomatic patients may experience sudden onset of dim and blurred vision, micropsia, metamorphopsia, decreased color vision, and central scotoma. ${ }^{5}$ Although the condition is normally unilateral, bilateral involvement have been reported in about $40 \%$ of cases. ${ }^{1,2}$ Further, CSCR typically occurs more frequently in young males than in females. ${ }^{3}$ Thus, males show a significantly higher mean annual incidence of CSCR than females, and the peak mean annual incidence occurs in men between 35 and 39 years. ${ }^{6}$ In most cases, CSCR is self-limiting and resolves spontaneously in 2-3 months, though usually without a complete recovery of visual function. ${ }^{3}$ Furthermore, some patients may suffer from persistent or recurrent
Psychiatric Clinics of the University of Basel, Center for Affective, Stress and Sleep Disorders, Wilhelm Klein-Strasse 27, 4012 Basel, Switzerland

$\mathrm{Tel}+4|6| 3255$ II4

Fax $+4|6| 3255513$

Email serge.brand@upkbs.ch 
CSCR with subsequent progressive loss of vision. ${ }^{7}$ Recurrent CSCR is associated with a variety of psychiatric disorders. ${ }^{7}$ Thus, the quality of life (QoL) of patients with CSCR may be compromised as a side effect of disturbing symptoms that occur during both acute and chronic phases of the disease. $^{3}$

Neither the etiology nor the pathophysiology of CSCR is completely understood. Nonetheless, a range of psychological and psychophysiological variables have been found to be associated with the occurrence of CSCR. For example, correlations of type A personality, systemic hypertension, and obstructive sleep apnea have been reported with CSCR disease. ${ }^{2,8}$ Further, almost half of patients with CSCR have been reported to use exogenous steroids. ${ }^{9}$ Other risk factors are pregnancy, use of antibiotics, antihistamines, alcohol, smoking, and respiratory diseases. ${ }^{10-16}$ Further, Conrad et $\mathrm{al}^{17}$ showed that patients with CSCR were more stressed as a result of inadequate coping strategies and a higher incidence of physical complaints.

Several studies have shown that patients with CSCR have higher scores for psychopathological symptoms when compared to healthy controls., ${ }^{3,17}$ Conrad et al ${ }^{17}$ found that CSCR patients had significantly higher emotional distress as measured by the Global Severity Index compared to healthy controls. This may reflect inadequate coping strategies, as indicated in higher levels of physical complaints in the patient group. ${ }^{17}$ Sahin et $\mathrm{al}^{3}$ found that CSCR patients had more marked psychological symptoms and poorer QoL than healthy control subjects. They also showed that the psychological status and QoL of CSCR patients varied with degree of loss of vision. ${ }^{3}$ Siguan and Aguilar ${ }^{5}$ found that patients with CSCR were more likely to display tendencies to schizophrenia (84\%), hysteria (83\%), depression $(75 \%)$, psychopathic deviance (67\%), and hypochondriasis (58\%) than control groups. Piskunowicz et al concluded that compared to healthy controls, patients with CSCR were more insecure, reported higher levels of frustration and higher levels of anticipatory anxiety. ${ }^{16}$ In another study, greater use of psychopharmacologic drugs was identified as an independent risk factor for the development of CSCR. ${ }^{6}$ In this study, not exposure to but intake of antianxiety drugs was predominantly observed in male participants.

Taken together, numerous studies show an association between the occurrence of CSCR and psychological and psychophysiological characteristics. Further, the study by Tsai et $\mathrm{al}^{6}$ indicated that CSCR may be associated with the use of antianxiety drugs, though, surprisingly, to the best of our knowledge, no study has yet examined the association between the occurrence of CSCR and anxiety. In this specific context, anxiety is understood as a psychobiological process with the following characteristics: emotions such as fears and worries; anticipation of the worst, irritability, feelings of tension, loss of interests, and inability to relax; cognitions including dysfunctional thoughts of not being able to cope with issues or catastrophizing; somatic complaints such as pains, aches, weakness, and flushes; physiological alterations such as gastrointestinal pain, high heart rate, respiratory symptoms, and genitourinary symptoms; and behavioral changes such as sighing and rapid respiration, hand tremor, fidgeting, and avoidance of situations which might reduce anxiety.

Given that CSCR is associated with dramatically impaired vision, a human being's most important sense, we anticipate that CSCR will be associated with anxiety and believe this link should be investigated. We also believe that if CSCR is associated with anxiety, then this offers a good starting point for psychotherapeutic treatment, with cognitive-behavioral therapies (CBT) having proved efficacious in the treatment of anxiety. Additionally, we note that CSCR is self-limiting and resolves spontaneously in 2-3 months. Accordingly, treating CSCR-related anxiety should be particularly efficacious. Finally, if patients are experiencing CSCR for a second time, they should be aware that the condition is generally fully reversible, that it resolves spontaneously within 2-3 months, and, consequently, they should experience less anxiety.

As regards the relation between anxiety and sex, a recent review ${ }^{18}$ concluded that female adults were twice as likely as males to develop or display anxiety disorders (see also ${ }^{16}$ ). However, to our knowledge, it is unclear to what extent females suffering CSCR will also report greater anxiety. Accordingly, a further aim of the present study was to explore to what extent anxiety scores differ between male and female patients with CSCR.

Based on the literature, the following two hypotheses were formulated. First, we expected higher anxiety scores in patients with CSCR when compared with healthy controls. Second, we expected lower anxiety scores in CSCR patients suffering from CSCR for a second time. We treated as exploratory the question of whether females with CSCR have higher anxiety scores than males with CSCR.

To address these research questions, a sample of Iranian patients with CSCR was assessed; a sex- and age-matched control group was assessed in parallel. Evidence concerning patients with CSCR has predominantly been gathered in Western countries; with the present study, we aimed to 
contribute to the research on CSCR with a non-Western sample.

\section{Materials and methods Procedure and samples}

A total of 30 patients (mean age: 36.10 years, standard deviation $=2.34 ; 43 \%$ female) suffering from CSCR were assessed in the Farshchian Hospital, Hamadan, Iran, between 2012 and 2013. Of these, 13 patients were suffering from CSCR for a second time. This sample therefore allowed exploration of anxiety as a function of number of occurrences of CSCR. In parallel, a control group was assessed (mean age: 35.90 years, standard deviation $=2.30$; $t[58]=0.34, P=0.74, d=0.087$; sex distribution: $\chi^{2}[\mathrm{~N}=60$, $d f=1]=0.28, P=0.60)$. These healthy controls were recruited via word-of-mouth-recommendation and via advertisements among staff members of the Farshchian Hospital. To avoid possible confounders, the control group was matched for sex, age, marital status, economic circumstances, educational level, and place of residence. Table 1 gives the demographic data and statistical comparisons between the three groups.

To gather demographic, CSCR-related and anxietyrelated data, the assessment of both patients and healthy controls involved a brief medical interview and medical analysis of CSCR, focusing on current and past CSCR (for patients only), and on anxiety. To assess CSCR, in addition to medical history records, an ophthalmologist not further involved in the study performed fluorescein angiography and optical coherence tomography. Further, unilateral or bilateral involvement and data on loss of visual acuity and duration were assessed. Patients with a first or second CSCR did not descriptively and statistically differ as regards sociodemographic variables, uni- versus bilateral involvement, loss of acuity of the current CSCR, and duration of CSCR (see Table 1). A trained psychologist not further involved in the study performed the brief interview to assess anxiety (see below). All participants were fully informed about the purpose of the study, and provided written informed consent. The study was approved by the Ethics Committee of the Hamadan University of Medical Sciences (Hamadan, Iran), and was conducted in accordance with the ethical standards laid down in the Declaration of Helsinki.

\section{Assessing severity of anxiety: experts' ratings with the Hamilton Anxiety Rating Scale (HAM-A)}

The Hamilton Anxiety Rating Scale (HAM-A) ${ }^{19}$ is a rating scale consisting of 14 items focusing on symptoms of anxiety. Specifically, experts ask patients about anxious mood (worries, anticipation of the worst, fearful anticipation, irritability), tension (feelings of tension, fatigability, startle response, moved to tears easily, trembling, feelings of restlessness, inability to relax), fears (of dark, of strangers, of being left alone, of animals, of traffic, of crowds), insomnia (difficulty falling asleep, broken sleep, unsatisfying sleep and fatigue on waking, dreams, nightmares, night terrors), intellectual difficulties (difficulty concentrating, poor memory), depressed mood (loss of interest, lack of pleasure in hobbies, depression, early waking, diurnal swing), somatic complaints (muscular pains and aches, twitching, stiffness, myoclonic jerks, grinding of teeth, unsteady voice, increased muscular tone), somatic complaints (sensory: tinnitus, blurring of vision, hot and cold flushes, feelings of weakness, pricking sensation), cardiovascular symptoms (tachycardia, palpitations, pain in chest, throbbing of vessels, fainting feelings, missing beat),

Table I Demographic data of patients suffering from idiopathic CSCR (first or second time) and healthy controls

\begin{tabular}{|c|c|c|c|c|}
\hline & \multicolumn{3}{|l|}{ Groups } & \multirow[t]{3}{*}{ Statistics } \\
\hline & CSCR: first time & CSCR: second time & Controls & \\
\hline & $\mathbf{N}=\mathbf{I 7}$ & $\mathbf{N}=\mathbf{I 3}$ & $\mathbf{N}=\mathbf{3 0}$ & \\
\hline Sex (female/male) & $8 / 9$ & $5 / 8$ & $11 / 19$ & $\chi^{2}(\mathrm{~N}=60, d f=2)=0.45, P=0.93$ \\
\hline Age (years) M (SD) & $36.06(2.38)$ & $36.06(2.38)$ & $35.90(2.28)$ & $F(2,59)=0.06 \mathrm{I}, P=0.94$ \\
\hline Civil status (single/married) & $10 / 7$ & $8 / 5$ & $20 / 10$ & $\chi^{2}(\mathrm{~N}=60, d f=2)=0.56, P=0.82$ \\
\hline Education (diploma/higher education) & $9 / 8$ & $8 / 5$ & $11 / 19$ & $\chi^{2}(\mathrm{~N}=60, d f=2)=0.45, P=0.89$ \\
\hline Employment (unemployed/employed) & $10 / 7$ & $7 / 6$ & $18 / 12$ & $\chi^{2}(\mathrm{~N}=60, d f=2)=0.89, P=0.65$ \\
\hline Residency (city/countryside) & $12 / 5$ & $13 / 0$ & $24 / 6$ & $\chi^{2}(\mathrm{~N}=60, d f=2)=0.34, P=0.78$ \\
\hline Income (below/above Imio Rials) & $2 / 15$ & $2 / 13$ & $3 / 27$ & $\chi^{2}(\mathrm{~N}=60, d f=2)=0.08, P=0.89$ \\
\hline Unilateral versus bilateral involvement & $11 / 6$ & $10 / 3$ & - & $\chi^{2}(\mathrm{~N}=30, d f=I)=1.01, P=79$ \\
\hline Duration of CSCR (months) M (SD) & $4.51(3.8)$ & $3.83(3.26)$ & & $t(28)=0.98, P=0.69$ \\
\hline Same eye as before: yes/no & - & $10 / 3$ & - & \\
\hline
\end{tabular}

Abbreviations: CSCR, central serous chorioretinopathy; $d f$, degrees of freedom. 
respiratory symptoms (pressure or constriction in the chest, choking feelings, sighing, dyspnea), gastrointestinal symptoms (difficulty in swallowing, wind abdominal pain, burning sensations, abdominal fullness, nausea, vomiting, stomach rumbling, looseness of bowels, loss of weight, constipation), genitourinary symptoms (frequency of micturition, urgency of micturition, amenorrhea, menorrhagia, development of frigidity, premature ejaculation, loss of libido, impotence), and autonomic symptoms (dry mouth, flushing, pallor, tendency to sweat, giddiness, tension headache, raising of hair). Experts also noted behavior at interview (fidgeting, restlessness, or pacing; tremor of hands; furrowed brow; strained face; sighing or rapid respiration; facial pallor; swallowing, etc).

Answers were scored on five-point Likert scales ranging from 0 (not present) to 4 (very severe), with higher sum scores reflecting more severe anxiety (range: $0-56$ points). The following categories were also defined: $<15$ points indicates mild anxiety, 15-28 points indicates moderate anxiety severity, 29-42 points indicates severe anxiety, and 43-56 points indicates extremely severe anxiety. Reliability, validity, and sensitivity of the tool have been reported for the Farsi version. ${ }^{20}$ (Cronbach's alpha of the present study: 0.91).

\section{Statistical analysis}

To compare the demographic data of patients and healthy controls, two Student's $t$-tests (age and duration of CSCR) and a series of $\chi^{2}$ tests were performed. CSCR-related information between patients with one or with two occurrences of CSCR were compared with $\chi^{2}$ tests and $t$-tests. Next, an analysis of variance was performed with the factors Group (patients with CSCR for the first time; patients with CSCR for the second time; healthy controls) and Sex (female versus male), and with Hamilton Anxiety Scale scores as the dependent variable. To explore the association between anxiety scores and CSCR-related data (duration, loss of acuity), Pearson's correlations were performed.
For $t$-tests, Cohen's $d$ effect sizes were reported with the following indices: $d<0.50$ : small effect, $d<0.79$ : medium effect, and $d>0.8$ : large effect. For analyses of variance, effect sizes were indicated with the partial eta-squared $\left(\eta^{2}\right)$, with $0.059 \geq \eta^{2} \geq 0.01$ indicating small, $0.139 \geq \eta^{2} \geq 0.06$ indicating medium, and $\eta^{2} \geq 0.14$ indicating large effect sizes.

\section{Results}

\section{Hamilton Anxiety Rating Scale scores}

Table 2 reports all descriptive and statistical information for the Hamilton Anxiety Scale scores, separately for Group (controls; patients: first CSCR; patients: second CSCR) and Sex (males versus females). Figure 1 shows the Hamilton Anxiety Rating Scale scores, separately for groups and sex.

Hamilton Anxiety Scale scores differed significantly between groups, with lower scores in the control group when compared to the two patient groups. There were no significant sex differences, and no significant Group by Sex interaction.

No statistically significant correlations were observed between the anxiety scores and the loss of acuity and duration of the current CSCR, the area of detachment, and the permeability and dilatation of choroid (ie, choroidal homeostasis; all rs $<0.11$, ps $>0.60)$.

\section{Discussion}

The key findings of the present study were that, compared to healthy controls, patients suffering from idiopathic CSCR experienced higher anxiety, that anxiety in patients with CSCR was higher irrespective of whether CSCR was experienced for the first or for the second time, and that anxiety scores did not differ between males and females. The present study adds to the literature on CSCR and psychological functioning in an important way in showing that the occurrence of CSCR was associated with anxiety.

Two hypotheses and one exploratory research question were formulated, and each of these is considered in turn.

Table 2 Overview of the descriptive and statistical statistics of Hamilton Anxiety Scale scores for the factors Group (patients with first- versus second-time idiopathic CSCR versus healthy controls) and Sex (females versus males)

\begin{tabular}{|c|c|c|c|c|c|c|}
\hline & \multicolumn{6}{|c|}{ Groups } \\
\hline & \multicolumn{2}{|c|}{ Patients: first CSCR } & \multicolumn{2}{|c|}{ Patients: second CSCR } & \multicolumn{2}{|c|}{ Controls } \\
\hline & $\mathbf{N}$ & 17 & $\mathbf{N}$ & 13 & $\mathbf{N}$ & 30 \\
\hline Females M (SD) & 8 & $29.25(11.39)$ & 5 & $30.80(9.26)$ & 11 & $10.60(4.67)$ \\
\hline Males M (SD) & 9 & $26.89(11.73)$ & 8 & $23.75(9.62)$ & 19 & $12.83(7.089)$ \\
\hline \multirow[t]{3}{*}{ Statistics } & \multicolumn{6}{|c|}{ Factor Group: $F(2,54)=25.47, P=0.000$, partial eta-squared $=0.485(L)$} \\
\hline & \multicolumn{6}{|c|}{ Factor Sex: $F(I, 54)=0.86, P=0.357$, partial eta-squared $=0.016(S)$} \\
\hline & \multicolumn{6}{|c|}{ Factor Group by Sex interaction: $F(2,54)=1.40, P=0.25$, partial eta-squared $=0.049(\mathrm{~S})$} \\
\hline
\end{tabular}

Note: Indices are means and standard deviations are in parentheses.

Abbreviations: CSCR, central serous chorioretinopathy; L, large effect size; S, small effect size. 


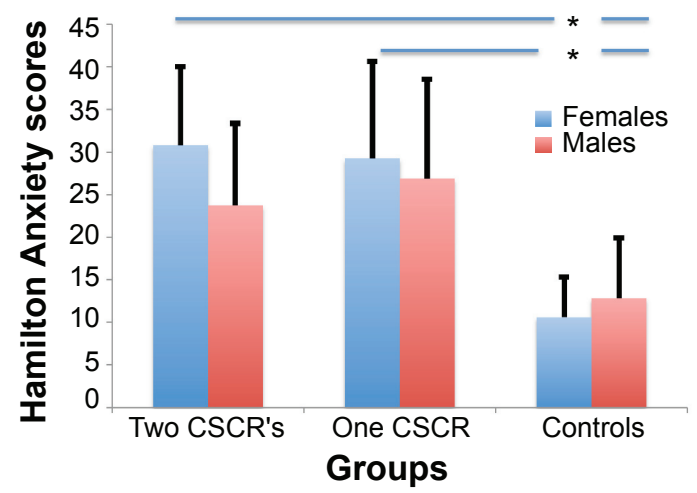

Figure I Hamilton Anxiety Scale scores for different groups separated by sex. Notes: Hamilton Anxiety Rating Scale scores differed statistically significantly (*) between healthy controls and patients with central serous chorioretinopathy, irrespective of whether patients suffered for the first or second time. No sex differences were observed. Bars are means; lines are standard deviations. Abbreviation: CSCR, central serous chorioretinopathy.

Our first hypothesis was that patients with CSCR would have higher scores for anxiety than healthy controls, and this hypothesis was fully confirmed. This result is consistent with the pattern of previous findings showing that occurrence of CSCR is associated with psychological issues. ${ }^{3,7,16,17}$ However, the present study adds to the current literature in an important new way; it is the first demonstration of a link between CSCR and the experience of anxiety. We believe this result is noteworthy for at least three reasons. First, if it is accepted that vision is the most important of human senses, it is not surprising that impairment of vision is associated with anxiety, that is, with the subjective experience of threat and danger. Second, given that vision is of overwhelming importance to human survival, ${ }^{21}$ it seems somewhat surprising that, to our knowledge, there has not previously been any investigation of the association between CSCR and anxiety. Third, indirect evidence for an association between anxiety and CSCR is provided by the observation that abuse of anxiolytics seems common among patients suffering from CSCR (Tsai et $\mathrm{al}^{6}$ ), suggesting that anxiety is a major concern among such patients. Overall, in our opinion, the present results might be considered an advance in the understanding of psychological processes and concerns among patients with CSCR.

Our second hypothesis was that anxiety would be lower among patients suffering from CSCR for the second time than among first-time sufferers. However, this hypothesis was not supported. This suggests that experiencing CSCR for a second time is not associated with an appreciation that CSCR is a transitory, treatable, and readily manageable impairment of vision. Unfortunately, the present data do not provide any deeper insight into patients' cognitive and emotional processes. Specifically, it was not clear why patients suffering from CSCR for a second time did not display more optimism and confidence about improvement in their condition, given that, in all patients suffering from CSCR for a second time, no residual or irreversible impairments of the ocular apparatus have been observed or reported in the ophthalmologic records. In other words, why had patients not learned from their first experience that suffering CSCR for a second time was no reason for fear? Again, the present data do not shed any light on the underlying cognitive-emotional mechanisms. Though highly speculative, we advance the following explanation. First, evidence shows that anxiety is a psychiatric disorder, which normally requires psychopharmacological and psychotherapeutic treatment. Accordingly, it is unlikely that anxiety would disappear without professional treatment and on the basis of "mere" experience. Second, by definition, anxiety is fueled by dysfunctional emotional and cognitive processes such as catastrophizing thinking and negative expectations, often built up over a long period of time. Accordingly, it is unlikely that experiencing CSCR for "only" the second time would be sufficient not to trigger anxiety again. Most importantly, this assumption is further supported by the observation that no meaningful associations between anxiety scores and loss of acuity and duration of the current CSCR, the area of detachment, and the permeability and dilatation of of choroid vessels (ie, choroidal homeostasis) could be observed. Third, if we take into account that patients suffering from CSCR might also have personality traits such as type A personality and low stress tolerance, ${ }^{16,17}$ the underlying common factor might be anxiety. Fourth, given that CSCR might also be associated with subsequent partial loss of vision, ${ }^{7}$ fear of losing vision when CSCR occurs for a second time is not surprising. Finally, given that the recurrence of CSCR might also be associated with impaired $\mathrm{QoL}^{3}$ and increased risk of suffering from psychiatric disorders, ${ }^{7}$ the persistence of anxiety is consistent with the cognitive-emotional framework surrounding impaired QoL and psychiatric disorders. In our view, several factors in combination are likely to ensure that anxiety is not lower when experiencing CSCR for a second time.

Last, we treated as exploratory the question as to whether female patients with CSCR would exhibit higher anxiety scores than male patients. It has been observed that, compared to adult males, adult females are one-and-a-half to two times more likely to suffer from anxiety. ${ }^{18}$ However, in the present study, we found no such difference. Accordingly, these results add to the current literature in a further important way by indicating that anxiety associated with CSCR is not sex-biased.

Despite the intriguing results, several limitations warn against overgeneralizing the results. First, the sample size 
was small, though the statistical indices focused on effect sizes, which are not sensitive to sample size. Second, data were gathered at one study center and a systematic bias in data collection and sample is therefore possible. Third, and most importantly, we considered only anxiety. Whereas assessing anxiety in patients with CSCR was the key focus of the present study, we are fully aware that anxiety might be a comorbid symptom of further underlying dysfunctional cognitive-emotional processes such as personality traits and psychiatric disorders. Future studies should therefore include a thorough psychiatric assessment. Fourth, in this respect, further unassessed but latent psychophysiological factors might have biased two or more dimensions in the same direction.

\section{Conclusion}

Among patients suffering from CSCR, anxiety is substantially higher than in healthy controls. Anxiety scores were not lower if CSCR was experienced for a second time, suggesting therefore that no psychophysiological adaptation occurs with repetition of CSCR.

\section{Acknowledgment}

We thank Nick Emler (University of Surrey, Surrey, UK) for proofreading the manuscript.

\section{Disclosure}

The authors report no conflicts of interest in this work.

\section{References}

1. Wang M, Munch IC, Hasler PW, Prünte C, Larsen M. Central serous chorioretinopathy. Acta Ophthalmol. 2008;86:126-145.

2. Liu DT, Fok AC, Chan W, et al. Central serous chorioretinopathy. In: Ryan SJ, Schachat AP, Wilkinson CP, Hinton DR, Sadda SR, Wiedemann P, editors. Retina. 5th ed. Elsevier Health Sciences; 2012:1292-1305.

3. Sahin A, Bez Y, Kaya MC, Türkcü FM, Sahin M, Yüksel H. Psychological distress and poor quality of life in patients with central serous chorioretinopathy. Semin Ophthalmol. 2014;29(2):73-76.

4. Regillo C, Holekamp N, Johnson MW, et al. Basic and Clinical Science Course-Retina and Vitreous. Singapore: LEO; 2012.

5. Siguan CS, Aguilar RN. Psychological profile of patients with central serous retinopathy. Philipp J Ophthalmol. 2014;39:16-20.
6. Tsai DC, Chen SJ, Huang CC, et al. Epidemiology of idiopathic central serous chorioretinopathy in Taiwan, 2001-2006: a population-based study. PLoS One. 2013;8(6):e66858.

7. Fok AC, Chan PP, Lam DS, Lai TY. Risk factors for recurrence of serous macular detachment in untreated patients with central serous chorioretinopathy. Ophthalmic Res. 2011;46(3):160-163.

8. Leveque TK, Yu L, Musch DC, Chervin RD, Zacks DN. Central serous chorioretinopathy and risk for obstructive sleep apnea. Sleep Breath. 2007;11(4):253-257.

9. Carvalho-Recchia CA, Yannuzzi LA, Negrão S, et al. Corticosteroids and central serous chorioretinopathy. Ophthalmology. 2002;109(10): 1834-1837.

10. Haimovici R, Koh S, Gagnon DR, Lehrfeld T, Wellik S; Central Serous Chorioretinopathy Case-Control Study Group. Risk factors for central serous chorioretinopathy: a case-control study. Ophthalmology. 2004;111(2):244-249.

11. Cunningham ET Jr, Alfred PR, Irvine AR. Central serous chorioretinopathy in patients with systemic lupus erythematosus. Ophthalmology. 1996;103(12):2081-2090.

12. Jampol LM, Weinreb R, Yannuzzi L. Involvement of corticosteroids and catecholamine in the pathogenesis of central serous chorioretinopathy: a rationale for new treatment strategies. Ophthalmology. 2002; 109(10):1765-1766.

13. Mansuetta CC, Mason JO 3rd, Swanner J, et al. An association between central serous chorioretinopathy and gastroesophageal reflux disease. Am J Ophthalmol. 2004;137(6):1096-1100.

14. Bouzas EA, Scott MH, Mastorakos G, Chrousos GP, Kaiser-Kupfer MI. Central serous chorioretinopathy in endogenous hypercortisolism. Arch Ophthalmol. 1993;111(9):1229-1233.

15. Spahn C, Wiek J, Burger T, Hansen L. Psychosomatic aspects in patients with central serous chorioretinopathy. Br J Ophthalmol. 2003; 87:704-708.

16. Piskunowicz M, Jaracz M, Lesiewska H, Malukiewicz G, BrozekPestka M, Borkowska A. Temperament profile in patients with central serous chorioretinopathy: a case-control study. Eur J Ophthalmol. 2014;24(3):392-395.

17. Conrad R, Geiser F, Kleiman A, Zur B, Karpawitz-Godt A. Temperament and character personality profile and illness-related stress in central serous chorioretinopathy. Scientific World Journal. 2014;2014: 631687.

18. Catuzzi JE, Beck KD. Anxiety vulnerability in women: a two-hit hypothesis. Exp Neurol. 2014;259:75-80.

19. Hamilton M. The assessment of anxiety states by rating. Br J Med Psychol. 1959;32:50-55.

20. Hossein Kaviani H, Mousavi AS. Psychometric properties of the Persian version of Beck Anxiety Inventory (BAI). Tehran University Medical Journal. 2008;66(2):136-140.

21. Kandel ER, Schwartz JH, Jessell TM. Principles of Neuroscience. 4th ed. New York: McGraw-Hill; 2000.
Neuropsychiatric Disease and Treatment

\section{Publish your work in this journal}

Neuropsychiatric Disease and Treatment is an international, peerreviewed journal of clinical therapeutics and pharmacology focusing on concise rapid reporting of clinical or pre-clinical studies on a range of neuropsychiatric and neurological disorders. This journal is indexed on PubMed Central, the 'PsycINFO' database and CAS,

\section{Dovepress}

and is the official journal of The International Neuropsychiatric Association (INA). The manuscript management system is completely online and includes a very quick and fair peer-review system, which is all easy to use. Visit http://www.dovepress.com/testimonials.php to read real quotes from published authors. 